\title{
Wideband Substrate Integrated Waveguide Antenna for Next-Generation mmWave Wireless Systems
}

\author{
Thomas Deckmyn ${ }^{1}$, Ad C.F. Reniers ${ }^{3}$, A. Bart Smolders ${ }^{3}$, Hendrik Rogier ${ }^{1}$, Dries Vande Ginste ${ }^{1}$, Sam Agneessens ${ }^{1,2}$ \\ ${ }^{1}$ Dept. of Information Technology - IDLab, Ghent University - imec, Ghent, Belgium, thomas.deckmyn@ugent.be \\ ${ }^{2}$ Centre for Microsystems Technology, Ghent University - imec, Ghent, Belgium \\ ${ }^{3}$ Faculty of Electrical Engineering, Eindhoven University of Technology, Eindhoven, The Netherlands
}

\begin{abstract}
A wideband substrate integrated waveguide (SIW) antenna topology is proposed for operation in the $60 \mathrm{GHz}$ band. It consists of two coupled quarter-mode and one halfmode SIW resonant cavities. This novel approach combines a significant bandwidth enhancement and a small footprint with compatibility to low-cost, standard PCB fabrication technology, making it excellently suited for 5G high-datarate wireless applications. Exploiting SIW technology, a high antennaplatform isolation is obtained, enabling high-density integration with active electronics without the risk of harmful coupling. An antenna that covers the entire $60 \mathrm{GHz}$ IEEE 802.11ad band is designed, with a measured fractional impedance bandwidth of $11.7 \%(7 \mathrm{GHz})$. The measured maximum gain and radiation efficiency of the prototype amount to more than $5.1 \mathrm{dBi}$ and $65 \%$, respectively, within the entire impedance bandwidth.
\end{abstract}

Index Terms-Substrate integrated waveguide (SIW) antenna, coupled resonators, half-mode SIW (HMSIW), quarter-mode SIW (QMSIW), wideband, $60 \mathrm{GHz}$.

\section{INTRODUCTION}

Requirements with respect to channel capacity have become ever more stringent in recent years, as the demand for broadband wireless applications is rapidly increasing. A prime example of this tendency is $5 \mathrm{G}$ mobile communication, where the end user is in constant need for higher data rates. On top of that, electronic devices, and, in particular, end user equipment, continuously decrease in size. Therefore, it is paramount to develop novel wideband antenna topologies with limited footprints and compatibility to standard manufacturing processes, as to facilitate easy and compact integration into end user equipment, such as mobile handsets.

The $7 \mathrm{GHz}$ of globally available and unlicensed frequency spectrum in the $60 \mathrm{GHz}$ IEEE 802.11 ad band offers the ideal opportunity to meet the strict requirements of $5 \mathrm{G}$ wireless communication systems, both in terms of channel capacity and number of interconnected users. The atmospheric absorption peak yields ideal conditions for highly secure, low interference and short distance communication between many wireless devices sharing the same spectrum [1]-[2].

Presently, Substrate Integrated Waveguide (SIW) technology is making a breakthrough in the mmWave research field, owing to its outstanding shielding capabilities, excellent loss performance and compatibility to standard printed circuit board (PCB) manufacturing processes [3]. Recent trends include antennas [4], filters and couplers for

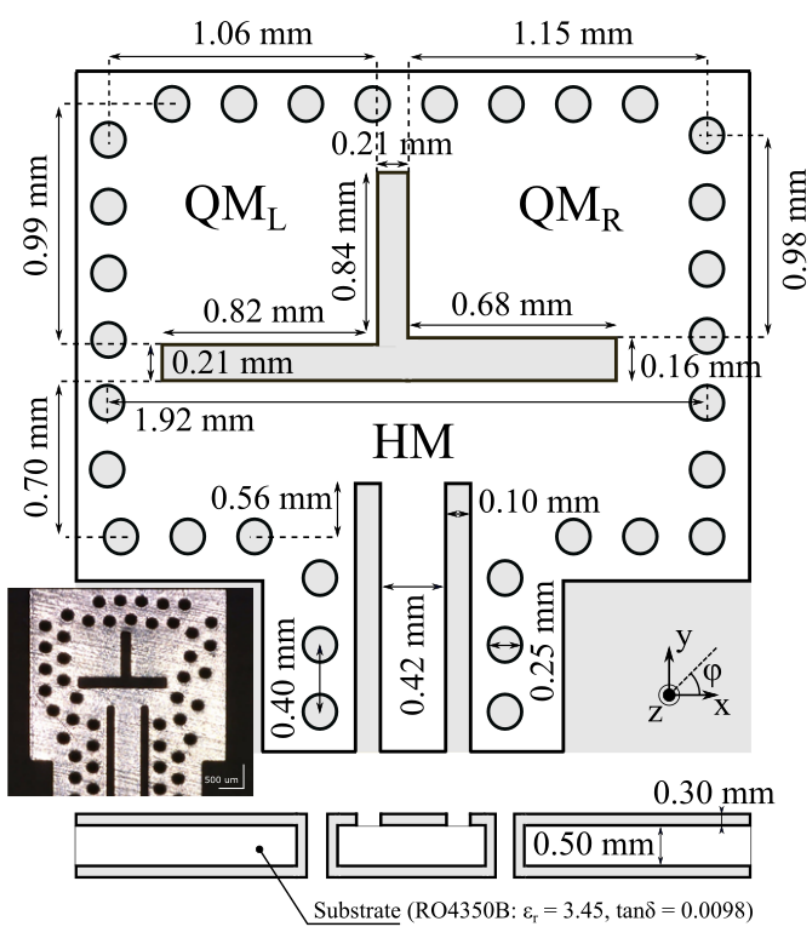

Fig. 1: Proposed antenna topology: three resonant SIW cavities, being two quarter-mode $\left(\mathrm{QM}_{\mathrm{L}}\right.$ and $\left.\mathrm{QM}_{\mathrm{R}}\right)$ and one half-mode $(\mathrm{HM})$, are incorporated into one single antenna element, yielding a significant bandwidth enhancement. Manufactured prototype as inset.

RF front-ends [5], MIMO systems [6] and beam steering [7]. Moreover, a rectangular SIW cavity can be miniaturized by bisecting the structure along virtual magnetic walls, as such obtaining half-mode (HMSIW) or quarter-mode (QMSIW) resonant cavities. The miniaturized SIW cavities almost completely retain the field distribution of the original SIW, and they exhibit excellent microwave performance [8]-[9].

A novel wideband SIW antenna topology is proposed in this paper. An innovative technique, based on three coupled, miniaturized resonators/antennas is exploited to achieve a significant bandwidth enhancement, all while maintaining an extremely small footprint and compatibility to low-cost, standard PCB manufacturing technologies. The distinct resonances of the coupled resonators are carefully distributed over the frequency band of interest, as such providing wideband behavior. These features make the proposed 
topology the ideal candidate for integration in $5 \mathrm{G}$ user equipment, such as mobile handsets.

SIW antenna designs for the $60 \mathrm{GHz}$ band, previously reported in literature, unavoidably made compromises to achieve sufficient bandwidth. SIW slot arrays have proven to yield high impedance bandwidths, but suffer from excessively large form factors [10]-[11]. Often, multi-layer PCB technology or low temperature co-fired ceramic (LTCC) needs to be exploited to realize air cavities [12] or thick substrates [13]-[14], resulting in a significant increase of complexity and manufacturing cost. Moreover, stacked patch antennas demonstrate large impedance bandwidths. Nevertheless, these topologies suffer from a fairly low antenna/platform isolation, making SIW technology the most viable choice for high-density integration in $5 \mathrm{G}$ mobile handsets.

\section{ANTENNA PRINCIPLE OF OPERATION}

The proposed $60 \mathrm{GHz}$ wideband antenna topology is depicted in Fig. 1. It consists of one half-mode (HMSIW) and two quarter-mode (QMSIW) SIW resonant cavities, combined into a single antenna element. Basically, this innovative topology combines three separate antenna elements into the footprint of a traditional half-wavelength SIW antenna, resulting in a substantial reduction of the form factor. The three miniaturized cavities in Fig. 1 are brought in close proximity, yielding three coupled resonating cavities. Owing to the coupling, mode bifurcation occurs. As such, the entire system, consisting of one HMSIW and two QMSIWs, exhibits three specific resonances, of which the frequencies are determined by the dimensions of the miniaturized cavities and the coupling between them.

In this design, the HMSIW is the feeding cavity, meaning that only the half-mode SIW is excited via an external feed line. Owing to the strong coupling, the QMSIWs are in turn excited by the HMSIW. Hence, they are in fact parasitic resonators. The antenna radiates via the inverted $\mathrm{T}$-slot separating the distinct miniaturized cavities, as shown in Fig. 1.

The first step in the design process consists of determining the dimensions of the HMSIW and the two QMSIW cavities, as to obtain three resonances in the desired frequency range. This is done according to

$f_{101, H M}=\frac{c}{\sqrt{2 \pi \varepsilon_{r, e f f}}} \sqrt{\left(\frac{\pi}{W_{e f f, H M}}\right)^{2}+\left(\frac{\pi}{2 L_{e f f, H M}}\right)^{2}}$,

for the HMSIW cavity, and

$f_{101, Q M}=\frac{c}{\sqrt{2 \pi \varepsilon_{r, e f f}}} \sqrt{\left(\frac{\pi}{2 W_{e f f, Q M}}\right)^{2}+\left(\frac{\pi}{2 L_{e f f, Q M}}\right)^{2}}$,

for the two QMSIWs, where $W_{\text {eff }}$ and $L_{\text {eff }}$ are the effective width and length of the resonant cavities, respectively, and $\varepsilon_{r, e f f}$ is the effective relative permittivity of the substrate material.

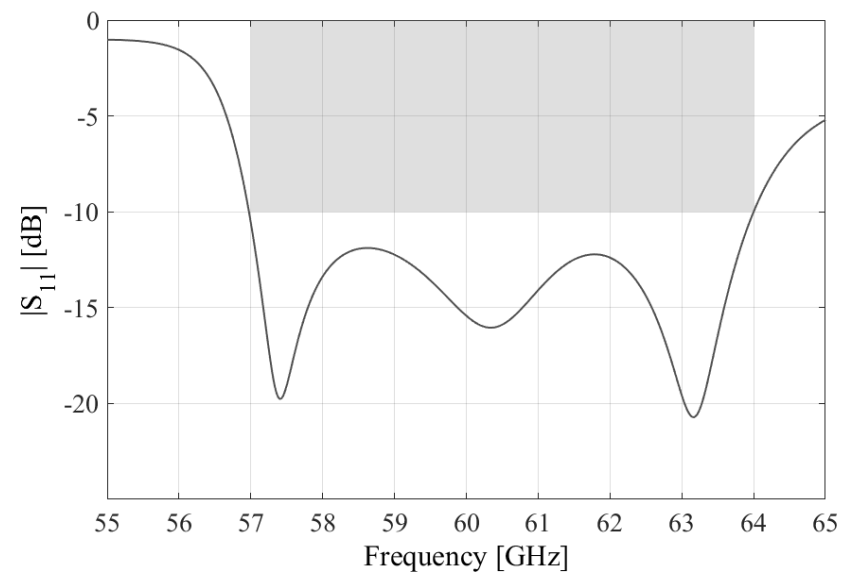

Fig. 2: Simulated reflection coefficient for the novel proposed topology. By distributing the three distinct resonances over the entire $60 \mathrm{GHz}$ band, a considerable bandwidth enhancement is obtained.

Accurate fine-tuning is performed in the second step, where the coupling is meticulously controlled by optimizing the dimensions of the inverted T-slot. In turn, this yields an optimal distribution of the resonances within the frequency range of interest and, consequently, a significant bandwidth enhancement. Moreover, three radiating elements are combined into a single antenna element with a footprint in the order of half a wavelength, without loss of performance. Both impedance bandwidth and form factor are drastically improved, without the need for a trade-off, which is, in fact, the prime novelty of this work.

The simulated reflection coefficient, presented in Fig. 2, clearly exhibits three distinct resonance peaks. An impedance bandwidth of $7 \mathrm{GHz}$, or $11.7 \%$, is achieved, covering the entire $60 \mathrm{GHz}$ IEEE $802.11 \mathrm{ad}$ band. The simulated electric field distribution inside the miniaturized cavities, at the three resonance frequencies, is depicted in Fig. 3. It is clear from Fig. 3 (a) that the E-field is dominant in the left QMSIW at 57.5 GHz, corresponding to the first resonance peak perceived in the reflection coefficient from Fig. 2. Likewise, the E-field at the second and third resonance is dominant in the right QMSIW and the HMSIW, respectively. The electric field distribution in the HMSIW and the two QMSIWs are equal to a half and a quarter of the field distribution in a full

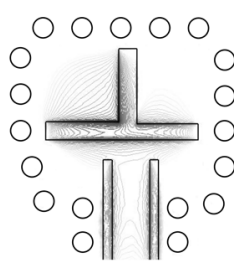

(a)

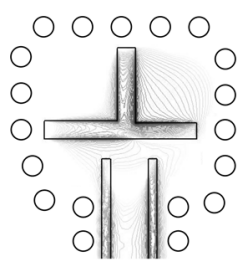

(b)

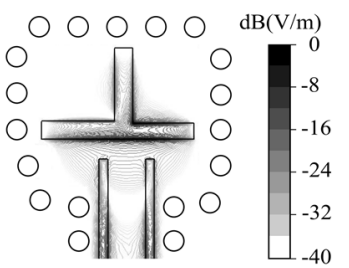

(c)
Fig. 3: Normalized electric field distribution inside the antenna, simulated at the three resonance frequencies: (a) $57.5 \mathrm{GHz}$, (b) $60.4 \mathrm{GHz}$ and (c) 63 $\mathrm{GHz}$. 
rectangular SIW cavity, respectively. This proves that the miniaturization technique can be applied without performance loss.

\section{EXPERIMENTAL VALIDATION}

The fabricated prototype is presented as an inset in Fig. 1. An additional row of via holes is added as compared to the simulation model. Nevertheless, this is merely a precaution and does not have an impact on the performance of the antenna. S-parameter measurements are conducted using a Keysight N5247A PNA-X Network Analyzer and $1.85 \mathrm{~mm}$ Southwest Microwave End-Launch connector assemblies. A custom Thru-Reflect-Line (TRL) calibration kit was developed and used to position the port reference plane closer to the antenna, thereby de-embedding both the feed line and the connector assembly.

The measured reflection coefficient is presented in Fig. 4 and corresponds very well with the simulated values. A -10 $\mathrm{dB}$ impedance bandwidth of $7 \mathrm{GHz}$, corresponding to a fractional bandwidth of $11.7 \%$, is achieved, as such covering the entire $60 \mathrm{GHz}$ IEEE $802.11 \mathrm{ad}$ band.

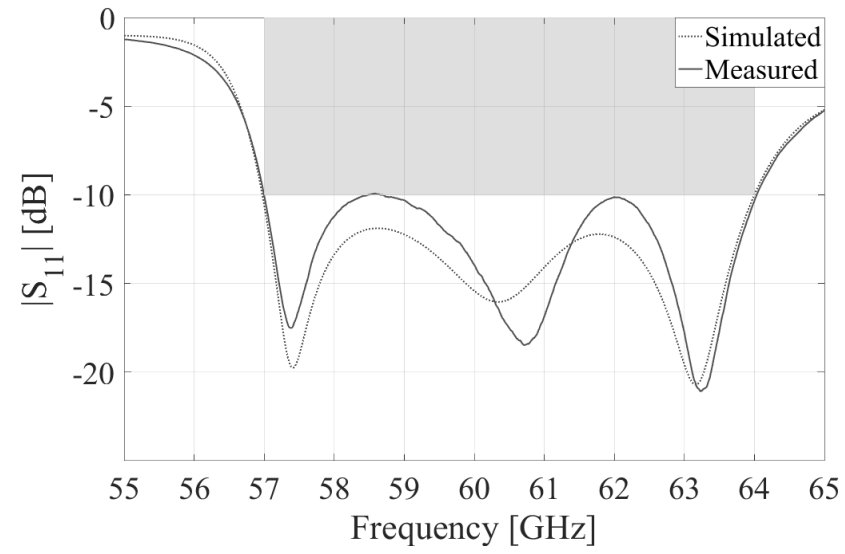

Fig. 4: Measured reflection coefficient of the fabricated prototype (solid line) versus simulated values (dotted line).

Cross sections of the measured and simulated gain patterns are presented in Fig. 5, for the resonance at 57.5 GHz in the left QMSIW, in Fig. 6, for the second resonance at 61 $\mathrm{GHz}$ in the right QMSIW, and in Fig. 7, for the third resonance at $63 \mathrm{GHz}$ in the HMSIW. As discussed in Section II, different cavity resonances are dominant at different frequencies. This results in frequency-dependent E-planes, accordingly defined as $\varphi=135^{\circ}, 45^{\circ}$ and $90^{\circ}$ for the resonance in the left QMSIW, right QMSIW and HMSIW, respectively. Likewise, the corresponding $\mathrm{H}$-planes are defined as $\varphi=45^{\circ}, 135^{\circ}$ and $0^{\circ}$. Clearly, there is a good agreement between the measured and simulated radiation patterns at all three resonance frequencies, both in the Eplanes and H-planes. The simulation model includes the measurement probe, as well as the mount for the antenna under test (AUT), as their influence on the radiation pattern is no longer negligible at mmWave frequencies. Doing so, all parasitic radiation, reflection and diffraction are accounted for, approximating the realistic measurement scenario as closely as possible.

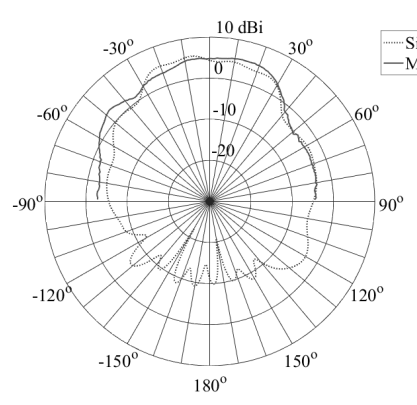

(a)

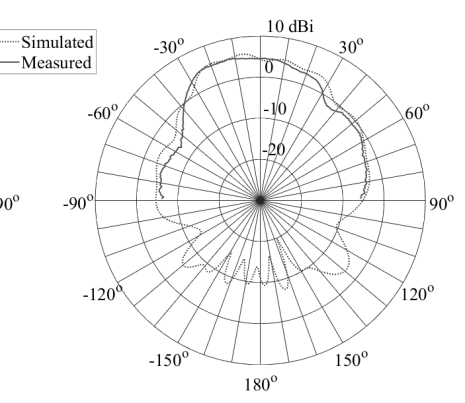

(b)
Fig. 5: Measured and simulated total gain radiation pattern at $57.5 \mathrm{GHz}$ (resonance in left QMSIW): (a) E-plane at $\varphi=135^{\circ}$, (b) H-plane at $\varphi=45^{\circ}$.

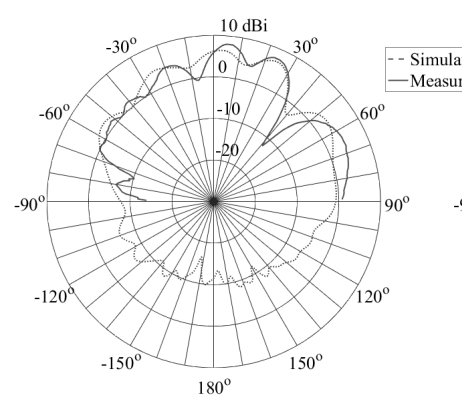

(a)

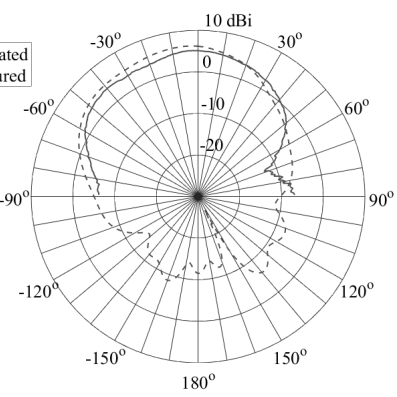

(b)
Fig. 6: Measured and simulated radiation pattern at $61 \mathrm{GHz}$ (resonance in right QMSIW): (a) E-plane at $\varphi=45^{\circ}$, (b) H-plane at $\varphi=135^{\circ}$.

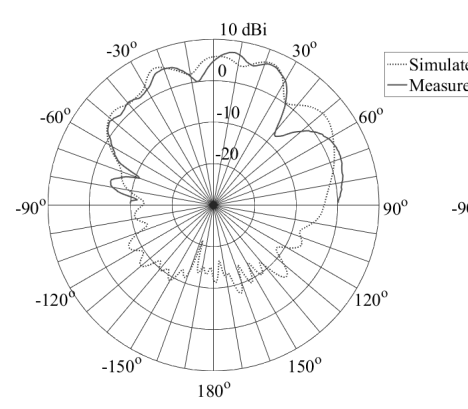

(a)

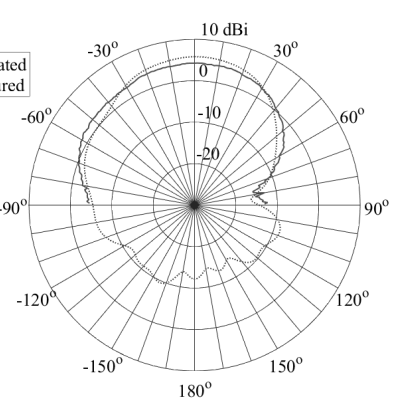

(b)
Fig. 7: Measured and simulated radiation pattern at $63 \mathrm{GHz}$ (resonance in HMSIW): (a) E-plane at $\varphi=90^{\circ}$, (b) H-plane at $\varphi=0^{\circ}$.

TABLE I. MEASURED AND Simulated BRoAdSide GaIN AND RAdiation EFFICIENCY AT THE THREE RESONANCE FREQUENCIES

\begin{tabular}{|l|c|c|c|c|}
\hline \multirow{2}{*}{ Frequency } & \multicolumn{2}{|c|}{ Gain } & \multicolumn{1}{c|}{ Radiation Efficiency } \\
\cline { 2 - 5 } & Meas. & Sim. & \multicolumn{1}{c|}{ Meas. } & \multicolumn{1}{c|}{ Sim. } \\
\hline $57.5 \mathrm{GHz}$ & $5.92 \mathrm{dBi}$ & $5.80 \mathrm{dBi}$ & $65.8 \%$ & $64.3 \%$ \\
\hline $61 \mathrm{GHz}$ & $6.32 \mathrm{dBi}$ & $6.45 \mathrm{dBi}$ & $67.7 \%$ & $69.3 \%$ \\
\hline $63 \mathrm{GHz}$ & $5.14 \mathrm{dBi}$ & $5.16 \mathrm{dBi}$ & $67.5 \%$ & $67.8 \%$ \\
\hline
\end{tabular}


The measured and simulated broadside gain values and radiation efficiencies are summarized in Table $\mathrm{I}$. The measured and simulated results correspond quite well. The measured broadside antenna gain is larger than $5.1 \mathrm{dBi}$ within the entire $7 \mathrm{GHz}$ impedance bandwidth, with a maximum of $6.32 \mathrm{dBi}$ at the resonance at $61 \mathrm{GHz}$ in the right QMSIW. As shown in Table I, the measured radiation efficiency is higher than $65 \%$ over the entire band, with a maximum of $67.7 \%$ at $61 \mathrm{GHz}$.

\section{CONCLUSION}

A novel wideband SIW antenna topology based on coupled resonators has been designed and validated for operation in the $60 \mathrm{GHz}$ IEEE $802.11 \mathrm{ad}$ band. Three tightly coupled radiating elements are combined into a single antenna with a footprint of about half a wavelength. The impedance bandwidth is significantly enhanced by carefully distributing their distinct resonances over the frequency band of operation. This large bandwidth is achieved without the need for a trade-off in terms of footprint or cost. The design is fully compatible with standard two-layer PCB manufacturing processes. A measured fractional impedance bandwidth of $11.7 \%(7 \mathrm{GHz})$ is obtained. The measured broadside antenna gain is larger than $5.1 \mathrm{dBi}$ over the entire impedance bandwidth. A measured radiation efficiency of more than $65 \%$ is obtained.

The shielding capabilities of the SIW technology confine the electromagnetic fields within the cavities, prohibiting propagation of surface waves. Hence, the novel HM- and QMSIW antenna is the ideal candidate for close integration in large antenna arrays or with active electronics for applications such as $5 \mathrm{G}$ mobile user equipment.

\section{ACKNOWLEDGMENT}

The authors would like to thank the ERC for the advanced grant 695495 "ATTO: A new concept for ultra-high capacity wireless networks". Part of this work was supported by BELSPO through the IAP Phase VII BESTCOM project.

\section{REFERENCES}

[1] P. Smulders, "Impact of regulations on feasible distance between 60 $\mathrm{GHz}$ devices," in 2010 Proceedings of the Fourth European Conference on Antennas and Propagation (EuCAP), 2010, pp. 1-4.

[2] A. Deshmukh and S. Bodhe, "Characterization of radio propagation at $60 \mathrm{GHz}$ channel," in First Asian Himalayas International Conference on Internet (AH-ICI), Nov 2009, pp. 1-8.

[3] M. Bozzi, A. Georgiadis and K. Wu, "Review of substrate-integrated waveguide circuits and antennas," IET Microwaves, Antennas \& Propagation, vol. 5, no. 8, pp. 909-920, June 62011.

[4] T. Deckmyn, S. Agneessens, A. C. F. Reniers, A. B. Smolders, M. Cauwe, D. Vande Ginste, H. Rogier, "A Novel $60 \mathrm{GHz}$ Wideband Coupled Half-Mode/Quarter-Mode Substrate Integrated Waveguide Antenna," IEEE Transactions on Antennas and Propagation, to be published, doi: 10.1109/TAP.2017.2760360.

[5] F. F. He, K. Wu, W. Hong, L. Han and X. P. Chen, "Low-Cost 60-GHz Smart Antenna Receiver Subsystem Based on Substrate Integrated
Waveguide Technology," IEEE Transactions on Microwave Theory and Techniques, vol. 60, no. 4, pp. 1156-1165, April 2012.

[6] S. Lemey, T. Castel, P. Van Torre, T. Vervust, J. Vanfleteren, P. Demeester, D. Vande Ginste, H. Rogier, "Threefold Rotationally Symmetric SIW Antenna Array for Ultra-Short-Range MIMO Communication," IEEE Transactions on Antennas and Propagation, vol. 64, no. 5, pp. 1689-1699, May 2016.

[7] N. Tiwari and T. R. Rao, "A switched beam antenna array with butler matrix network using substrate integrated waveguide technology for 60 GHz communications," 2015 International Conference on Advances in Computing, Communications and Informatics (ICACCI), Kochi, 2015, pp. 2152-2157.

[8] C. Jin, R. Li, A. Alphones and X. Bao, "Quarter-Mode Substrate Integrated Waveguide and Its Application to Antennas Design," IEEE Transactions on Antennas and Propagation, vol. 61, no. 6, pp. 2921 2928, June 2013.

[9] Q. Wu, H. Wang, C. Yu and W. Hong, "Low-Profile Circularly Polarized Cavity-Backed Antennas Using SIW Techniques," IEEE Transactions on Antennas and Propagation, vol. 64, no. 7, pp. 28322839, July 2016.

[10] S. Liao, P. Chen, P. Wu, K. M. Shum and Q. Xue, "Substrate-Integrated Waveguide-Based 60-GHz Resonant Slotted Waveguide Arrays With Wide Impedance Bandwidth and High Gain," IEEE Transactions on Antennas and Propagation, vol. 63, no. 7, pp. 2922-2931, July 2015.

[11] J. Pourahmadazar and T. A. Denidni, "High Gain Substrate Integrated Waveguide Resonant Slot Antenna Array for mm-Wave Band Radio," 2015 IEEE International Conference on Ubiquitous Wireless Broadband (ICUWB), Montreal, QC, 2015, pp. 1-4.

[12] S. B. Yeap, Z. N. Chen and X. Qing, "Gain-Enhanced 60-GHz LTCC Antenna Array With Open Air Cavities," IEEE Transactions on Antennas and Propagation, vol. 59, no. 9, pp. 3470-3473, Sept. 2011.

[13] H. Jin, W. Che, K. S. Chin, G. Shen, W. Yang and Q. Xue, "60-GHz LTCC Differential-Fed Patch Antenna Array With High Gain by Using Soft-Surface Structures," in IEEE Transactions on Antennas and Propagation, vol. 65, no. 1, pp. 206-216, Jan. 2017.

[14] S. Liao and Q. Xue, "Dual Polarized Planar Aperture Antenna on LTCC for $60-\mathrm{GHz}$ Antenna-in-Package Applications," in IEEE Transactions on Antennas and Propagation, vol. 65, no. 1, pp. 63-70, Jan. 2017. 